\title{
Chaotic and periodic motions of a cyclic bus induced by speedup
}

\author{
Takashi Nagatani* \\ Department of Mechanical Engineering, Shizuoka University, Hamamatsu 432-8561, Japan
}

(Received 3 June 2002; published 9 October 2002)

\begin{abstract}
We study the effect of speedup on the dynamical behavior of a single cyclic bus in a bus system with many bus stops. We present a nonlinear-map model of a cyclic bus to take into account the speedup. When the cyclic bus is delayed, the bus speeds up to retrieve the delay. It is found that the cyclic bus exhibits chaotic motion with increasing speedup. The chaotic motion depends on both speedup and the number of bus stops. Also, it is shown that the dynamical transition between the chaotic and periodic motions occurs with the increase of bus stops. The dependence of the recurrence time (one period) and Liapunov exponent on both speedup and the number of bus stops is calculated for distinct dynamic states. It is shown that the speedup has a significant effect on the bus motion. For a piecewise linear-map model, the cyclic bus does not exhibit the chaotic motion but a complex oscillatory motion with multiple periods occurs.
\end{abstract}

DOI: 10.1103/PhysRevE.66.046103

PACS number(s): 89.40.+k, 05.45.-a, 82.40.Bj

\section{INTRODUCTION}

Recently, transportation problems have attracted much attention in the field of nonequilibrium statistical mechanics [1-4]. The jamming transitions of traffic flow have been investigated by using various models [5-15]. The dynamical phase transitions are very much similar to the conventional phase transitions and critical phenomena even if the system is far from equilibrium [9].

Very recently, bus route models with multiple buses have been formulated by models similar to the traffic flow [1621]. It has been found that the bunching transition between an inhomogeneous jammed phase and a homogeneous phase occurs with increasing buses. The bunching transition is definitely different from the jamming transitions of traffic flow.

In the system of a single cyclic bus on a circular route with many bus stops, a bus interacts with passengers waiting at bus stops [22]. With the increase of awaiting passengers at bus stops, a bus slows down, since it takes more time for awaiting passengers to board the bus. Then, the recurrence time (one period) converges or diverges with the increase of rotations. The cyclic bus exhibits a dynamical transition between the delay and schedule-time phases. The interaction between buses does not exist in the single-cyclic-bus problem but the dynamical phase transition occurs. The delay transition depends on the number of bus stops, the bus speed, and the number of awaiting passengers.

If the cyclic bus slows down, the bus will speed up in order to operate it on a schedule time. The operator may be able to overcome the bus delay by the speeding up. It is little known how the speedup affects the bus behavior. The bus behavior is governed by the dynamics of nonlinear map [23]. The delay transition is determined by the properties of the attractors. The bus exhibits such complex behaviors as chaotic and multiple periodic motions by the variation of the speedup and the number of bus stops.

In this paper, we study the dynamical behavior of the cyclic bus induced by the speedup when the bus slows down.

\footnotetext{
*Email address: tmtnaga@ipc.shizuoka.ac.jp
}

We present the nonlinear-map model to describe the bus dynamics. We investigate the dynamics of the nonlinear map. We show that the dynamical transitions to complex motion occur by changing the speedup and the number of bus stops. We calculate the Liapunov exponent for various dynamical states. We show that chaotic motion occurs with the increase of speedup.

\section{MODEL}

We present the model of a cyclic bus on a circular route. There are $M$ bus stops on the circular route. A cyclic bus starts at bus stop $m=1$ and moves around the route. The bus always stops at all the bus stops. If the recurrence time (one period) is less than the prescribed limit, the bus moves with a regular speed. When the recurrence time is larger than the prescribed limit, the bus speeds up and moves with higher speed. The model is defined on a finite one-dimensional lattice with a periodic boundary condition. Each lattice site is labeled by a number $m$ running from 1 to $M$. A site represents a bus stop. The distance between a bus stop $m-1$ and its next bus stop $m$ is defined by $L_{m}$. The average speed of the bus between a bus stop and its next bus stop at rotation $n$ is defined by $V(m, n)$. The arrival time $t(m, n+1)$ at bus stop $m$ at the $(n+1)$ th rotation is given by the summation of the stopping time (for new passengers to board the bus) and the moving time between a bus stop and the next bus stop over the bus stops $M$. One obtains the following equation:

$$
\begin{aligned}
t(m, n+1)= & t(m, n)+\sum_{l=1}^{m-1} F(\Delta t(l, n+1))+\sum_{l=1}^{m-1} \frac{L_{l}}{V(l, n+1)} \\
& +\sum_{l=m}^{M} F(\Delta t(l, n))+\sum_{l=m}^{M} \frac{L_{l}}{V(l, n)}
\end{aligned}
$$

where the function $F$ is the stopping time at a bus stop for new passengers to board the bus and the recurrence time $\Delta t(m, n)$ at bus stop $m$ at rotation $n$ is defined by $\Delta t(m, n)$ $=t(m, n)-t(m, n-1)$. 
The awaiting passengers at a bus stop increase with the recurrence time. The number of awaiting passengers at bus stop $m$ at the $n$th rotation is proportional to the recurrence time $\Delta t(m, n) . \mu$ is the rate of passengers arriving at the bus stop, so $\mu \Delta t(m, n)$ is the number of passengers that have arrived since the bus left the stop. The parameter $\kappa$ is the time it takes one passenger to board the bus, and so $\kappa \mu \Delta t(m, n)$ is the amount of time needed to board all the passengers. The boarding time increases with the increase of awaiting passengers.

However, when the awaiting passengers at a bus stop increase, the passengers form a queue. Then, in addition to boarding time $\kappa \mu \Delta t(m, n)$, one should take into account the time it takes one passenger to move from a position in the queue to the entrance of the bus. The moving time is given by $x / \nu_{q}$, where $x$ is the position of passenger in the queue and $\nu_{q}$ is the mean velocity of passenger. If the length of the queue is $L_{q}$, the moving time is given by $L_{q}^{2} / 2 \nu_{q}$. Since the length of the queue is proportional to the number of awaiting passengers, the moving time is proportional to the square of the recurrence time, $[\Delta t(m, n)]^{2}$. Therefore, the stopping time for new passengers to board the bus is given by $F(\Delta t(m, n))=\kappa \mu \Delta t(m, n)+[\zeta \mu \Delta t(m, n)]^{2} / 2 \nu_{q}$, where $\zeta$ is the space between passengers in the queue.

Generally, awaiting passengers at a bus stop interact each other and the human behavior will become more complex than the above. We assume that the boarding time is proportional to the power of the number of awaiting passengers:

$$
F(\Delta t(m, n))=\gamma_{0} \Delta t(m, n)^{\alpha},
$$

where $\gamma_{0}$ is the coefficient of proportionality and $1 \leqslant \alpha \leqslant 2$. On dividing time by the characteristic time $L / V_{0}$, one obtains $M$ simultaneous equations for the dimensionless recurrence time:

$$
\begin{aligned}
\Delta T(m, n+1)= & \sum_{l=1}^{m-1} \gamma \Delta T(l, n+1)^{\alpha}+\sum_{l=1}^{M} \gamma \Delta T(l, n)^{\alpha} \\
& +\frac{V_{0}}{V(n)},
\end{aligned}
$$

where $\Delta T(m, n)=V_{0} \Delta t(m, n) / L, \quad \gamma=\gamma_{0}\left(V_{0} / L\right)^{1-\alpha}$, route length $L=\sum_{l=1}^{M} L_{l}$, and $V(n)=\left(\sum_{l=1}^{M} V(l, n)\right) / M$ is the average velocity over a cycle.

We approximate Eq. (3) by a simple nonlinear map. The recurrence time $\Delta T(m, n)$ at bus stop $m$ is replaced by an effective (or mean) recurrence time $\Delta T(n)$. The $M$ simultaneous equations reduce to a nonlinear equation. One obtains the following nonlinear map of a single variable:

$$
\Delta T(n+1)=M \gamma \Delta T(n)^{\alpha}+V_{0} / V(n) .
$$

Map (4) presents a good approximation for Eq. (3) [22]. Figure 1 shows the plots of map (4) for (a) $\nu=V(n) / V_{0}$ $=1.0$ and (b) $\nu=V(n) / V_{0}=2.0$, where $\alpha=2.0, \gamma=0.02$, and $M=15$. Map (a) has no fixed points. Map (b) has two fixed points: 0.613 and 2.72 . The fixed point 0.613 is stable and

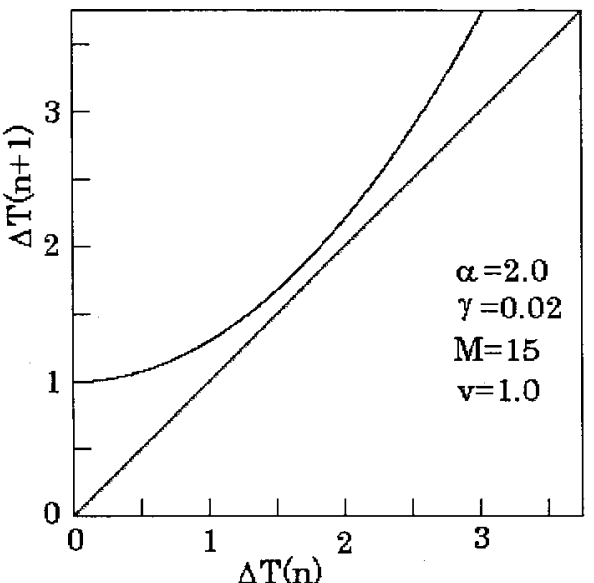

(a)

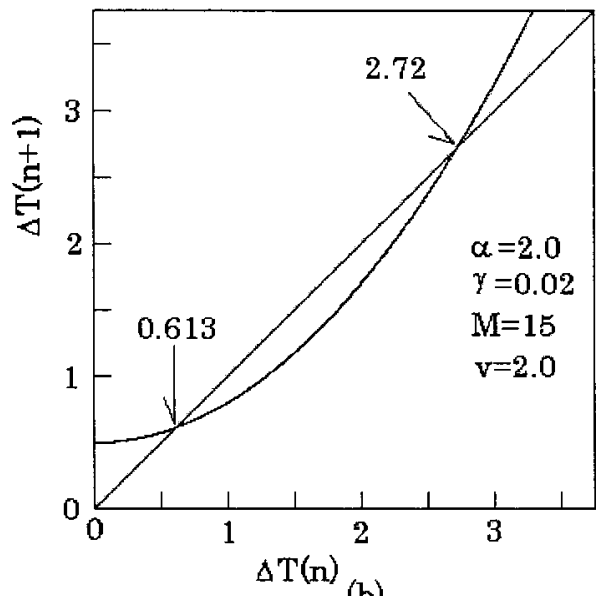

(b)

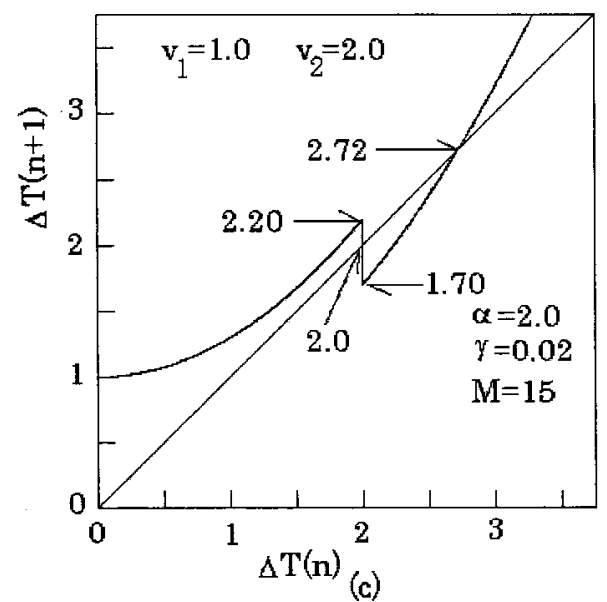

FIG. 1. Plots of map (4) for (a) $\nu=V(n) / V_{0}=1.0$ and (b) $\nu$ $=V(n) / V_{0}=20$, where $\alpha=2.0, \gamma=0.02$, and $M=15$. Map (a) has no fixed points. Map (b) has two fixed points: 0.613 and 2.72. (c) Plot of Eqs. (5) and (6) for $\nu_{1}=1.0$ and $\nu_{2}=2.0$, where $T_{\text {limit }}$ 2.0. The map has two unstable fixed points: 2.0 and 2.72 .

the fixed point 2.72 is unstable. The basin of attraction for the stable fixed point is given by $\Delta T(0)<2.72$.

If the recurrence time is larger than the prescribed limit $T_{\text {limit }}$, the bus moves with the high speed $\nu_{2}$. Otherwise, the bus moves with the low speed $\nu_{1}\left(\nu_{1}<\nu_{2}\right)$. The following map is obtained: 


$$
\begin{aligned}
& \Delta T(n+1)=M \gamma \Delta T(n)^{\alpha}+1 / \nu_{1} \quad \text { when } \Delta T(n) \leqslant T_{\text {limit }}, \\
& \Delta T(n+1)=M \gamma \Delta T(n)^{\alpha}+1 / \nu_{2} \quad \text { when } \Delta T(n)>T_{\text {limit }} .
\end{aligned}
$$

Figure 1(c) shows the plot of Eqs. (5) and (6) for $\nu_{1}=1.0$ and $\nu_{2}=2.0$, where $T_{\text {limit }}=2.0, \alpha=2.0, \gamma=0.02$, and $M$ $=15$. The map has two unstable fixed points: 2.0 and 2.72 . The basin of attraction for the fixed point 2.0 agrees nearly with that of map (b) but the dynamics is definitely different from map (b). It will be expected that the bus behavior exhibits chaotic or periodic motions near the fixed point 2.0.

The case of $\alpha=1.0$ presents the piecewise linear map [23]. First, we consider the piecewise nonlinear map of $\alpha$ $=2.0$, and then the piecewise linear map of $\alpha=1.0$.

\section{CHAOTIC AND PERIODIC MOTIONS}

We study the bus behavior by iterations of Eqs. (5) and (6). Figure 2 shows the plots of the recurrence time $\Delta T(n)$ against rotation number $n$ for initial values ranging over 0 $<\Delta T(0) \leqslant 3.0$, where $T_{\text {limit }}=2.0, \alpha=2.0, \gamma=0.02$, and $M$ $=15$. The diagrams (a) $-(\mathrm{c})$ indicate, respectively, the flow in the parameter space $(n, \Delta T(n))$ for the maps of Figs. 1(a), 1(b), and 1(c). For the map of Fig. 1(a), the recurrence time $\Delta T(n)$ diverges with the increase of rotation $n$ even if the bus starts from any initial value. The bus delays more and more. For the map of Fig. 1(b), when the initial value $\Delta T(0)$ is less than the unstable fixed point, the recurrence time $\Delta T(n)$ converges to the stable fixed point 0.613 with the increase of rotation $n$. Otherwise, the recurrence time diverges. For $\Delta T(0)<2.72$, the bus moves with a constant period, i.e., on the scheduled time. For $\Delta T(0)>2.72$, the bus delays more and more. Therefore, the dynamical transition between the delay and schedule-time phases occurs at $\Delta T(0)=2.72$. For the map of Fig. 1(c), the recurrence time exhibits the irregular behavior or diverges with the increase of rotation $n$.

We calculate the variation of the recurrence time by the increase of rotation to study the dynamical transitions to the irregular motions. Figure 3(a) shows the plot of the values of recurrence time $\Delta T(n)$ against the high speed $\nu_{2}$ from sufficiently large rotation, $n=100$ to $n=300$, when $\Delta T(0)$ $=2.0$, where $\nu_{1}=1.0, T_{\text {limit }}=2.0, \alpha=2.0, \gamma=0.02$, and $M$ $=15$. Figure 3(b) shows an enlargement Fig. 3(a) for $\nu_{2}$ $=1.5-1.9$. If the high speed $\nu_{2}$ is less than 1.33 , the recurrence time diverges with increasing rotation $n$. When high speed $\nu_{2}$ is higher than 1.33, the recurrence time takes irregular values. The dynamical transition from the divergence to the irregular motion occurs at the critical point $\nu_{2, c}$ $=1.33$. The critical point is given by such a point that the value of recurrence time (6) at $\Delta T(n)=T_{\text {limit }}=2.0$ for the high speed equals $T_{\text {limit }}=2.0$ [see Fig. 1(c)]:

$$
\Delta T_{\text {limit }}=M \gamma \Delta T_{\text {limit }}{ }^{\alpha}+1 / \nu_{2} .
$$

From Eq. (7), one obtains $\nu_{2, c}=1.33$ for $\alpha=2.0, \gamma=0.02$, and $M=15$. This value is consistent with that obtained from
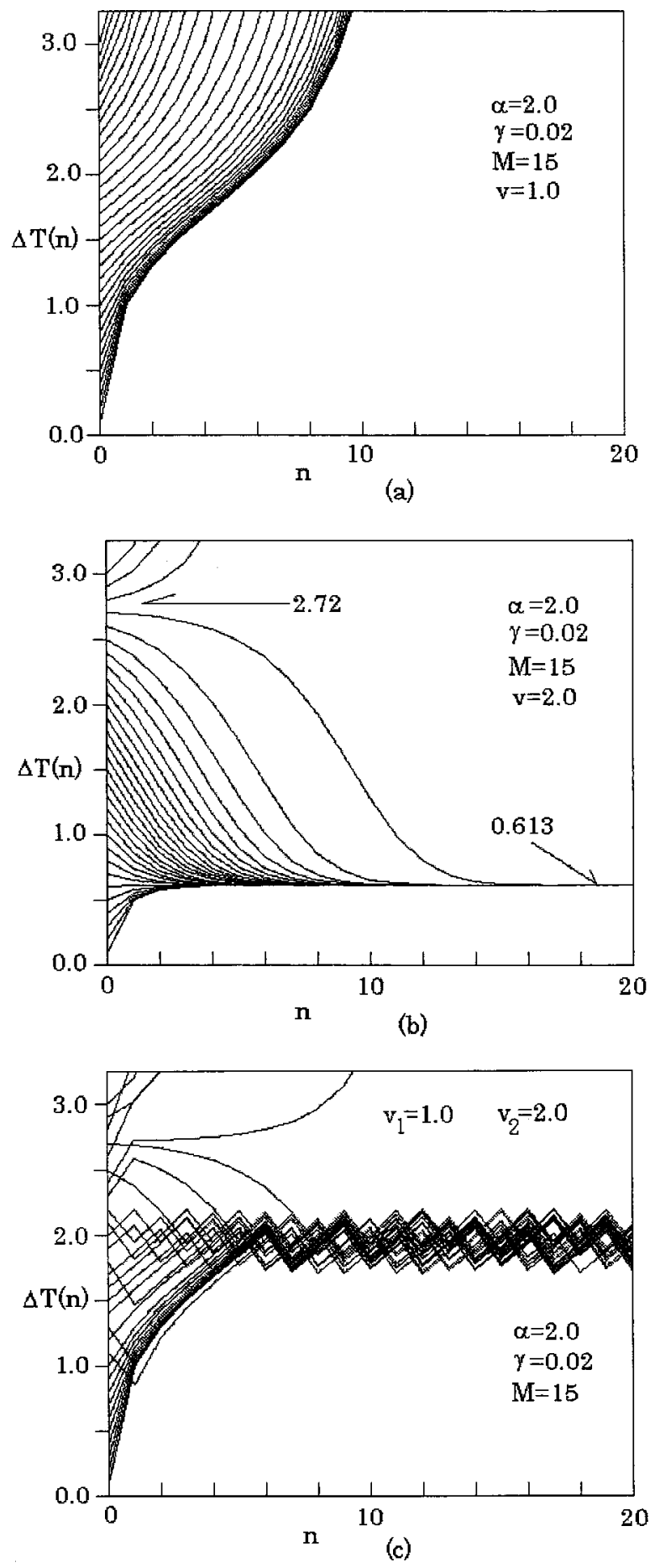

FIG. 2. Plots of the recurrence time $\Delta T(n)$ against rotation number $n$ for initial values ranging over $0<\Delta T(0) \leqslant 3.0$, where $T_{\text {limit }}=2.0, \alpha=2.0, \gamma=0.02$, and $M=15$. Diagrams (a)-(c) indicate, respectively, the flow in the parameter space $(n, \Delta T(n))$ for the maps of Figs. 1(a), 1(b), and 1(c).

the iteration. The maximum and minimum values $\Delta T_{\max }$ and $\Delta T_{\min }$ of the recurrence time are given, respectively,

$$
\begin{aligned}
& \Delta T_{\max }=M \gamma \Delta T_{\text {limit }}{ }^{\alpha}+1 / \nu_{1}, \\
& \Delta T_{\min }=M \gamma \Delta T_{\text {limit }}{ }^{\alpha}+1 / \nu_{2} .
\end{aligned}
$$



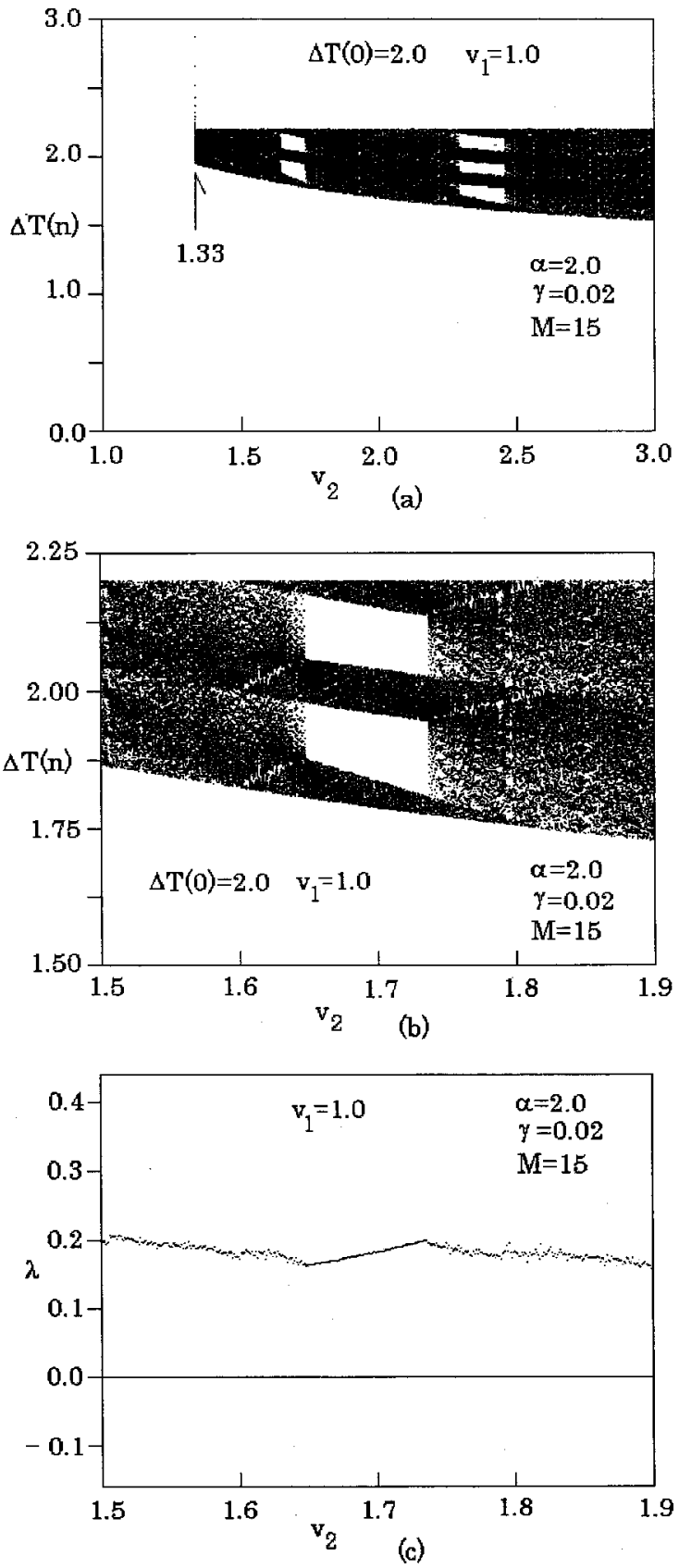

FIG. 3. Bus behavior of $M=15$. (a) Plot of the values of recurrence time $\Delta T(n)$ against the high speed $\nu_{2}$ from sufficiently large rotation $n=100$ to $n=300$ when $\Delta T(0)=2.0$, where $\nu_{1}=1.0$, $T_{\text {limit }}=2.0, \alpha=2.0$, and $\gamma=0.02$. (b) Enlargement of (a) for $\nu_{2}$ 1.5-1.9. (c) Plot of the Liapunov exponent against high speed $\nu_{2}$ for the same values of parameters as (b). The Liapunov exponent is positive.

For $\nu_{1}=1.0, \nu_{2}=2.0, \quad \alpha=2.0, \quad \gamma=0.02$, and $M=15$, the maximum and minimum values obtained from Eqs. (8) and (9) agree with those obtained from the iteration.

Figure 3(c) shows the plot of the Liapunov exponent against high speed $\nu_{2}$ for the same values of parameters in Fig. 3(b). The Liapunov exponent is positive. Therefore, the irregular behavior of recurrence time exhibits the chaotic motion of the cyclic bus when the high speed $\nu_{2}$ is higher than the critical value $\nu_{2, c}=1.33$.
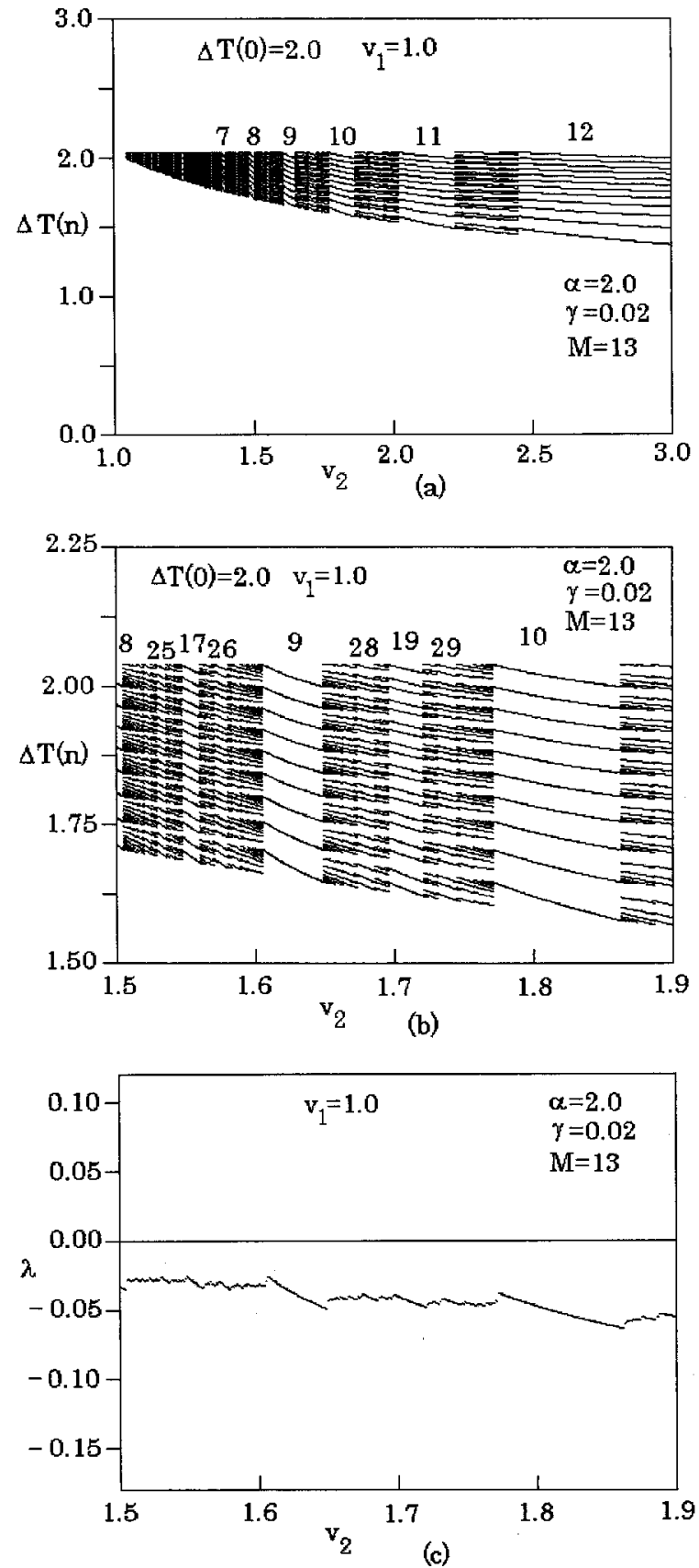

FIG. 4. Bus behavior of $M=13$. (a) Plot of the values of recurrence time $\Delta T(n)$ against the high speed $\nu_{2}$ from sufficiently large rotation $n=100$ to $n=300$ when $\Delta T(0)=2.0$, where $\nu_{1}=1.0$, $T_{\text {limit }}=2.0, \alpha=2.0$, and $\gamma=0.02$. (b) Enlargement of (a) for $\nu_{2}$ $=1.5-1.9$. (c) Plot of the Liapunov exponent against high speed $\nu_{2}$ for the same values of parameters as (b). The Liapunov exponent is negative.

We study the bus behavior when the bus stops $M=15$ reduces to $M=13$. Figure 4(a) shows the plot of the values of recurrence time $\Delta T(n)$ against the high speed $\nu_{2}$ from rotation $n=100$ to $n=300$ for $M=13$ when $\Delta T(0)=2.0$, where $\nu_{1}=1.0, T_{\text {limit }}=2.0, \alpha=2.0$, and $\gamma=0.02$. The values of parameters are the same as those of Fig. 3(a) except for $M$. Figure 4(b) shows an enlargement of Fig. 4(a) for $\nu_{2}$ $=1.5-1.9$. If the high speed $\nu_{2}$ is less than 1.042, the recur- 

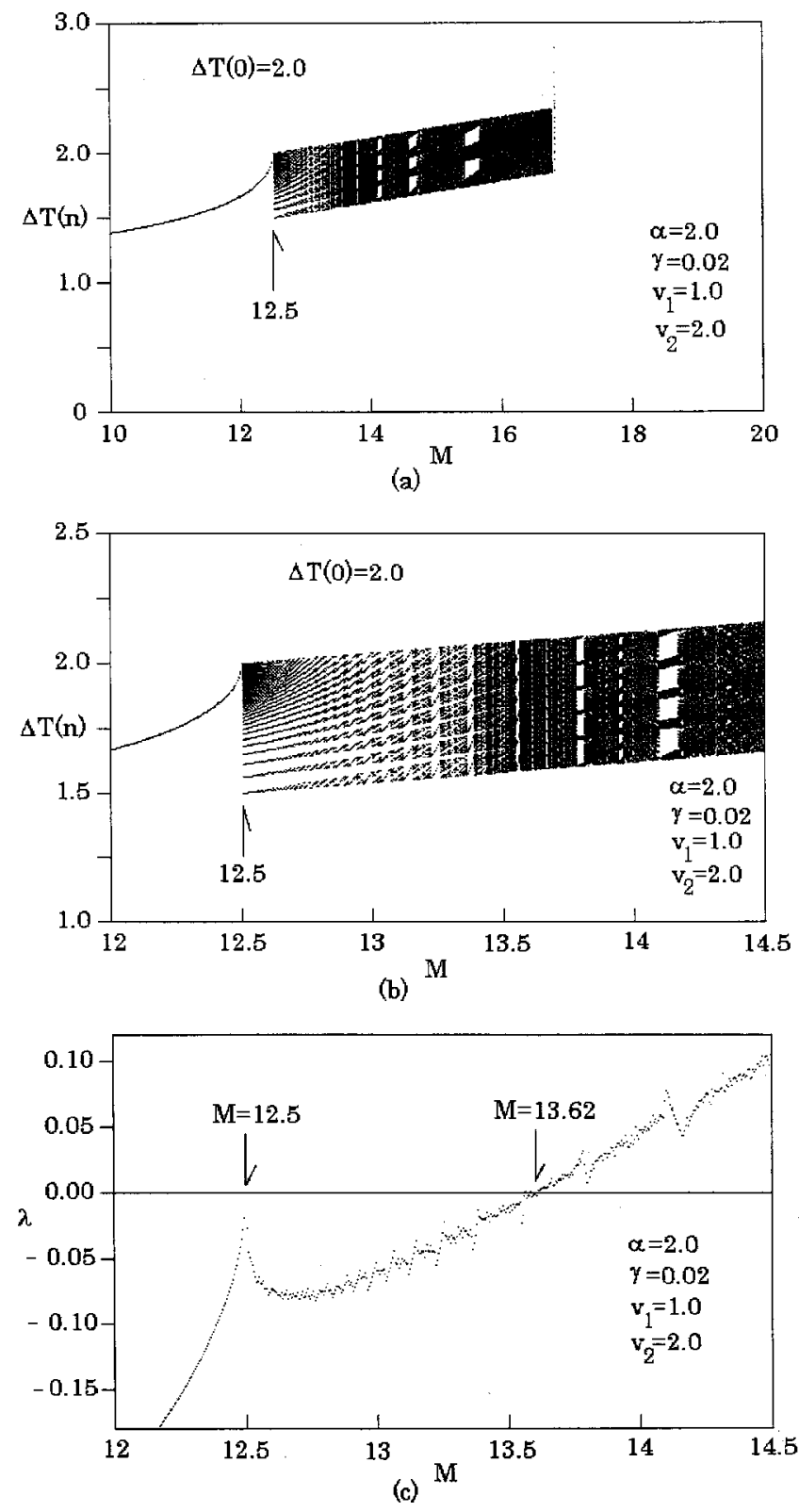

FIG. 5. (a) Plot of the values of recurrence time $\Delta T(n)$ against the number $M$ of bus stops from rotation $n=100$ to $n=300$ when $\Delta T(0)=2.0$, where $\nu_{1}=1.0, \nu_{2}=1.0, T_{\text {limit }}=2.0, \alpha=2.0$, and $\gamma$ $=0.02$. (b) Enlargement of (a) between $M=12$ and $M=14.5$. (c) Plot of the Liapunov exponent against bus stop number $M$ for the same values of parameters as (b).

rence time diverges with increasing rotation $n$. When high speed $\nu_{2}$ is higher than 1.042, the recurrence time takes periodic values. The dynamical transition from the divergence to the periodic motion occurs at the critical point $\nu_{2, c}$ $=1.042$. The critical point is obtained from Eq. (7). The periods range from 2 to values larger than 39. The periodic motion exhibits very complex bifurcations. The periods are shown as 8, 25, 17, 26, 9, 28,..., 10 in Fig. 4(b). The motion of period 19 appears between periods 9 and 10. The motion of period 29 appears between periods 19 and 10 . Thus, the motion of period $j+k$ occurs between periods $j$ and $k$. Figure 4(c) shows the plot of the Liapunov exponent against high

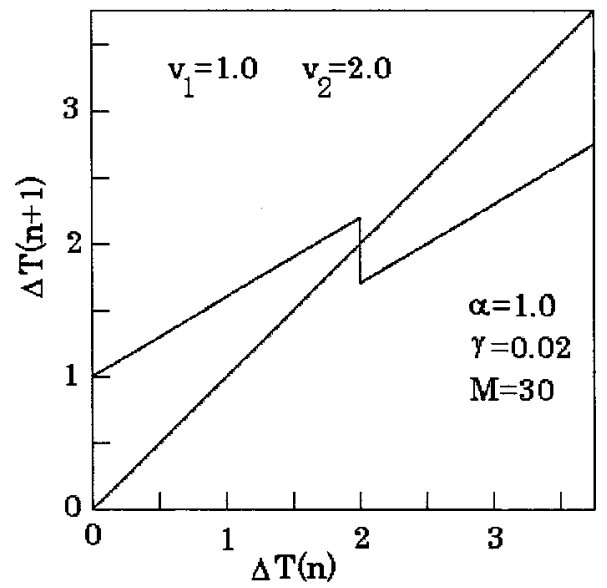

FIG. 6. Plot of Eqs. (5) and (6) for $\alpha=1.0$, where $\nu_{1}=1.0, \nu_{2}$ $=2.0, T_{\text {limit }}=2.0, \gamma=0.02$, and $M=30$. This map has only an unstable fixed point at $T_{\text {limit }}=2.0$.

speed $\nu_{2}$ for the same values of parameters in Fig. 4(b). The Liapunov exponent is negative. Therefore, it is confirmed that the motion of the cyclic bus is periodic when the high speed $\nu_{2}$ is higher than the critical value $\nu_{2, c}=1.042$.

We study the bus behavior by varying the number $M$ of bus stops under such a condition that the bus speeds up from $\nu_{1}=1.0$ to $\nu_{2}=2.0$. Figure 5(a) shows the plot of the values of recurrence time $\Delta T(n)$ against the number $M$ of bus stops from rotation $n=100$ to $n=300$ when $\Delta T(0)=2.0$, where $\nu_{1}=1.0, \nu_{2}=1.0, T_{\text {limit }}=2.0, \alpha=2.0$, and $\gamma=0.02$. Figure 5(b) shows an enlargement of Fig. 5(a) between $M=12$ and $M=14.5$. Figure 5(c) shows the plot of the Liapunov exponent against $M$ for the same values of parameters in Fig. 5 (b). When the number of bus stops is less than $M=12.5$, the recurrence time converges to the stable fixed point of the map with the increase of rotation $n$. At $M=12.5$, the dynamical transition from the schedule-time phase (convergence) to the periodic motion occurs. At $M=13.62$, the Liapunov exponent changes from a negative value to a positive value. The dynamical transition from the periodic motion (through the bifurcation to various periods) to the chaotic motion occurs at $M=13.62$. Furthermore, at $M=16.79$, the chaotic motion changes to the divergence of recurrence time. Thus, the chaotic motion of a cyclic bus appears for $M$ $=13.62-16.79$.

We study the case of $\alpha=1.0$. When the value of $\Delta T_{\min }$ given by Eq. (9) is higher than $T_{\text {limit }}$, the map (5) and (6) has a stable fixed point. If the drop of the map at $T_{\text {limit }}$ intersects the linear line $\Delta T(n+1)=\Delta T(n)$, the map has an unstable fixed point at $T_{\text {limit }}$. Then, it is expected that the bus should behave periodically or chaotically. Figure 6 shows the plot of Eqs. (5) and (6) for $\nu_{1}=1.0$ and $\nu_{2}=2.0$, where $T_{\text {limit }}=2.0$, $\alpha=1.0, \gamma=0.02$, and $M=30$. This map has only an unstable fixed point at $T_{\text {limit }}=2.0$. Figure 7 shows the plot of the recurrence time $\Delta T(n)$ against rotation $n$ for initial values ranging over $0<\Delta T(0) \leqslant 3.0$ for the map of Fig. 6. This exhibits a periodic motion of period 2. Figure 8 shows the plot of the values of recurrence time $\Delta T(n)$ against the high speed $\nu_{2}$ from sufficiently large rotation, $n=100$ to $n$ $=300$, when $\Delta T(0)=2.0$, where $\nu_{1}=1.0, T_{\text {limit }}=2.0, \alpha$ 


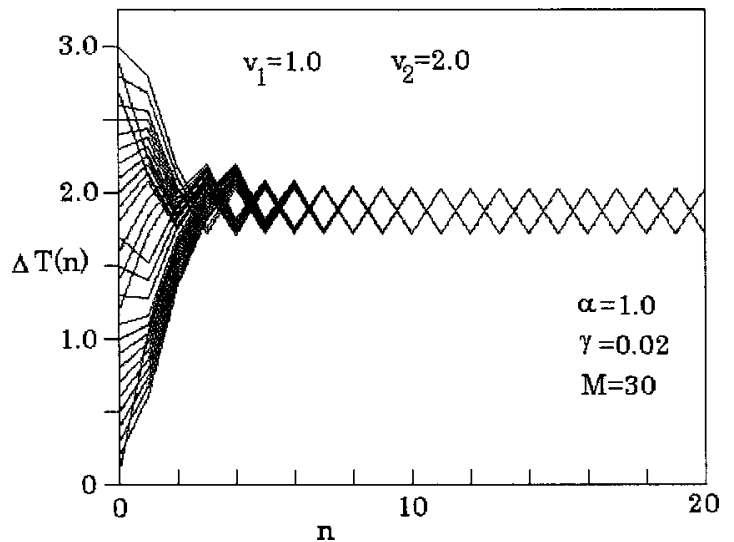

FIG. 7. Plot of the recurrence time $\Delta T(n)$ against rotation $n$ for initial values ranging over $0<\Delta T(0) \leqslant 3.0$ for the map of Fig. 6 . This exhibits the periodic motion of period 2 .

$=1.0, \gamma=0.02$, and $M=30$. If the high speed $\nu_{2}$ is less than 1.25 , the recurrence time converges to the stable fixed point and the bus moves on the scheduled time given by the stable fixed point. When the high speed $\nu_{2}$ is higher than 1.25 , the recurrence time takes periodic values after a sufficiently large rotation. At $\nu_{2, c}=1.25$, the dynamical transition from the schedule-time phase (convergence) to the periodic motion occurs. The motions of periods $2,3,4$, and 5 are observed.

For $\alpha=1.0$, we study the bus behavior by varying the number $M$ of bus stops under such a condition that the bus speeds up from $\nu_{1}=1.0$ to $\nu_{2}=2.0$. Figure 9 shows the plot of the values of recurrence time $\Delta T(n)$ against the number $M$ of bus stops from rotation $n=100$ to $n=300$ when $\Delta T(0)=2.0$, where $\nu_{1}=1.0, \nu_{2}=1.0, T_{\text {limit }}=2.0, \alpha=1.0$, and $\gamma=0.02$. At $M_{1, c}=25$, the dynamical transition from the schedule-time phase to the periodic motion occurs with the increase of the number $M$ of bus stops. By furthermore increasing $M$, the periodic motion changes to the schedule-time phase at $M_{2, c}=37.5$. The transition point $M_{1, c}$ is determined by

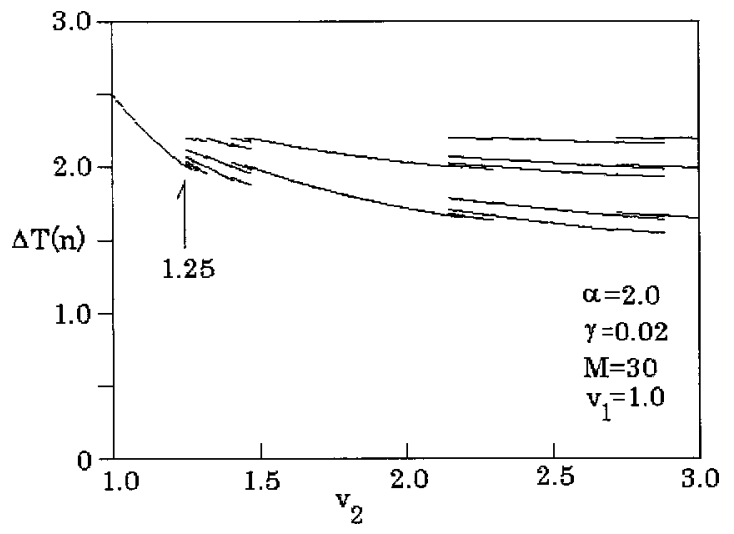

FIG. 8. Plot of the values of recurrence time $\Delta T(n)$ against the high speed $\nu_{2}$ from sufficiently large rotation $n=100$ to $n=300$ for $\alpha=1.0$ when $\Delta T(0)=2.0$, where $\nu_{1}=1.0, T_{\text {limit }}=2.0, \gamma=0.02$, and $M=30$.

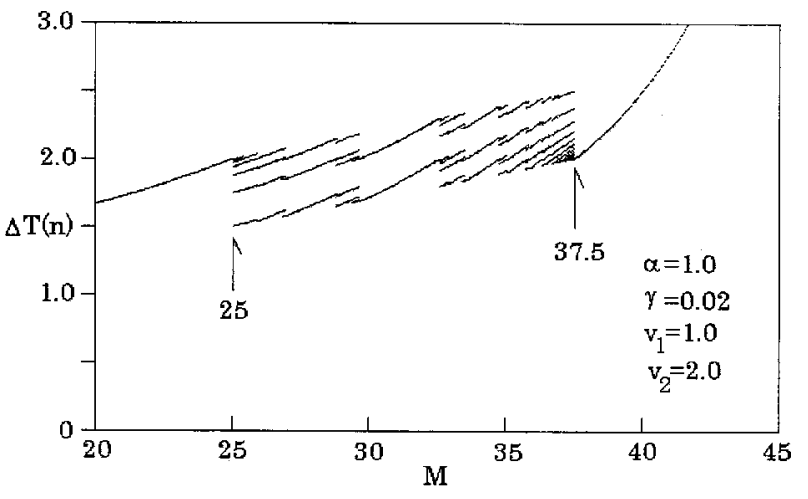

FIG. 9. Plot of the values of recurrence time $\Delta T(n)$ against the number $M$ of bus stops from rotation $n=100$ to $n=300$ for $\alpha$ $=1.0$ when $\Delta T(0)=2.0$, where $\nu_{1}=1.0, \nu_{2}=1.0, T_{\text {limit }}=2.0$, and $\gamma=0.02$.

$$
T_{\text {limit }}=M_{1, c} \gamma T_{\text {limit }}+1 / \nu_{1} \text {. }
$$

We obtain $M_{1, c}=25$ from Eq. (10). This value agrees with that obtained from iteration. The transition point $M_{2, c}$ is determined by

$$
T_{\text {limit }}=M_{2, c} \gamma T_{\text {limit }}+1 / \nu_{2} \text {. }
$$

We obtain $M_{2, c}=37.5$ from Eq. (11). This value also agrees with that obtained from iteration. The chaotic motion does not appear for $\alpha=1.0$ but the oscillatory motion with multiple periods occurs. Thus, the bus behavior is determined by the dynamical properties of the map (5) and (6).

\section{SUMMARY}

We have presented the nonlinear-map model of a single cyclic bus to take into account the speedup in a cyclic bus system. We have studied the effect of speedup on the dynamical behavior of a cyclic bus. The cyclic bus interacts with awaiting passengers at a bus stop. The bus is delayed with the increase of awaiting passengers. Then, the delayed bus speeds up to overcome the delay. As a result, the cyclic bus exhibits the complex behavior and dynamical transitions. We have shown that the bus behavior depends strongly on the speedup and the number of bus stops. We have found that the cyclic bus exhibits chaotic and periodic motions. We have also shown that the bus dynamics is determined by the properties of the fixed points of the nonlinear map. Finally, we have found that there are four distinct dynamical states: (1) the schedule-time phase (convergence of the recurrent time), (2) the delay phase (divergence of the recurrent time), (3) the chaotic motion, and (4) the multiply periodic motions. The transitions to the distinct dynamical states depend on the degree of speedup.

We have shown that the piecewise linear-map model ( $\alpha$ $=1$ ) does not exhibit chaotic motion, but complex oscillatory motions with multiple periods occur. 
[1] D. Chowdhury, L. Santen, and A. Schadscheider, Phys. Rep. 329, 199 (2000).

[2] D. Helbing, Rev. Mod. Phys. 73, 1067 (2001).

[3] B. S. Kerner, Netw. Spatial Econ. 1, 35 (2001).

[4] Traffic and Granular Flow '99, edited by D. Helbing, H. J. Herrmann, M. Schreckenberg, and D. E. Wolf (Springer, Heidelberg, 2000).

[5] K. Nagel and M. Schreckenberg, J. Phys. I 2, 2221 (1992).

[6] M. Bando, K. Hasebe, A. Nakayama, A. Shibata, and Y. Sugiyama, Phys. Rev. E 51, 1035 (1995).

[7] E. Ben-Naim, P. L. Krapivsky, and S. Redner, Phys. Rev. E 50, 822 (1994).

[8] O. Biham, A. A. Middleton, and D. Levine, Phys. Rev. A 46, R6124 (1992).

[9] T. Nagatani, Phys. Rev. E 58, 4271 (1998).

[10] K. Nagel, D. E. Wolf, P. Wagner, and P. Simon, Phys. Rev. E 58, 1425 (1998).

[11] E. Tomer, L. Safonov, and S. Havlin, Phys. Rev. Lett. 84, 382
(2000).

[12] M. Treiber, A. Hennecke, and D. Helbing, Phys. Rev. E 62, 1805 (2000).

[13] H. K. Lee, H.-W. Lee, and D. Kim, Phys. Rev. E 64, 056126 (2001).

[14] W. Knospe, L. Santen, A. Schadschneider, and M. Schreckenberg, e-print cond-mat/0203347.

[15] B. S. Kerner, Phys. Rev. E 65, 046138 (2002).

[16] O. J. O'loan, M. R. Evans, and M. E. Cates, Phys. Rev. E 58, 1404 (1998).

[17] D. Chowdhury and R. C. Desai, Eur. Phys. J. B 15, 375 (2000).

[18] T. Nagatani, Physica A 287, 302 (2000).

[19] T. Nagatani, Phys. Rev. E 63, 036116 (2001).

[20] T. Nagatani, Physica A 296, 320 (2001).

[21] H. J. C. Huijberts, Physica A 308, 489 (2002).

[22] T. Nagatani, Physica A 297, 260 (2001).

[23] H. G. Schuster, Deterministic Chaos (VCH, Weinheim, 1988). 